\title{
Clinical governance in mental health services
}

\section{A view from the 'shop floor'}

\author{
Jim Isherwood
}

In this paper, I explain the strategy for achieving clinical governance within the trust's mental health directorate, and describe the role that clinicians and managers would like the chief executive to play in helping us to achieve our targets.

\section{Clinical what?}

Quality has become the watchword of the NHS, or so the Government would like to think. It is not difficult to see why an emphasis on quality is important. Poor practice has often gone unchecked to the astonishment of the public, devastation of the victims, and the dissatisfaction of silent fellow clinicians who continue to provide a sound service. The challenge facing us is to "guarantee fair access and high quality to patients wherever they live".

\section{The road to quality}

The trust is too large and its activities too diverse for the implementation of clinical governance to be the responsibility of one senior clinician. History of change in the NHS demonstrates the need for innovation to be developed and owned at grassroots level. In my own speciality (forensic psychiatry), attempts to establish therapeutic and academic centres in the early 1960 s were doomed to failure because of the lack of central funding and absence of charismatic enthusiasts at local level (Snowden, 1990). Low secure units have progressed more successfully due to local product champions (Department of Health \& Home Office, 1992).

The York Mental Health Directorate decided to create a programme for quality that would utilise resources from the trust's Clinical Effectiveness Department. The existing clinical audit coordination group for mental health assumed responsibility for leading this process and became the Mental Health Clinical Governance Committee (CGC).

\section{The CGC}

This committee develops proposals for mental health clinical audit and submits bids to the Clinical Standards Executive (see p. 712, Fig. 1) for resources to conduct some of these. It is chaired by the mental health services business manager and includes a lead clinician (J.I.), service managers from the elderly, adult and children's departments and support managers with responsibility for quality and information. A member of the Clinical Effectiveness Department sits on the group to ensure that our clinical governance priorities are supported.

The CGC is influential in four activities related to clinical governance. These are the assimilation of evidence of best practice and the demands of stakeholders, setting of standards, measurement of the quality of service delivery and distribution of quality information. The last is self-explanatory. Regular reports of all aspects of quality must be made available to the trust board, hospital managers and senior managers. Quality (or lack of it) can no longer be a secret and key external stakeholders will also demand access to this information. The other three activities of the CGC are expanded below.

\section{Assimilation of evidence}

Many professionals in the NHS welcome the opportunity to improve care to patients and are driven to seek and deliver safer and more effective treatments. Consequently, it is essential that evidence of best practice is distributed to clinical teams. The trust's Clinical Effectiveness Department has this role, and the CGC acts as a focal point for distribution within the mental health directorate. The group also commissions advice locally so that national demands are combined with the needs of the local population in determining the priorities for our service.

In mental health the demands of the National Service Framework will influence the quality programme, but many standards are already explicit in mental health legislation and the Code 
of Practice. Proponents of change include the Mental Health Act Commission, the Royal College of Psychiatrists, Health Authorities and the Zito Trust (Sheppard, 1996) to name but a few. Service users and carers are being surveyed for their views and Mind, through its advocacy service, is encouraged to contribute.

\section{Standard setting}

The Mental Health Directorate has a wellestablished procedure for investigating untoward patient-related incidents, which are routinely reported to senior managers who discuss the level of inquiry required with the medical director of the trust. The national profusion of inquiries has generated criticism, but inquiries are essential (Grounds, 1997).

In York, a review of such incidents has provided ammunition to develop services where resources are inadequate and has comforted families and staff when no blame has been found. Where mistakes have been made, these must not be swept under the carpet. Failure to learn from error is the stuff of major inquiries and a sign of a sick organisation. The recent Fallon investigation at Ashworth Hospital provides a reminder of this (Fallon et al, 1999). Audit of the recommendations from our own series of adverse incidents will prove whether the prescribed actions have occurred.

Evaluation of adverse incidents is one of several priorities for clinical audit. We also expect the Clinical Governance Support Service run by the College to provide opportunities for participating in regional and national audit. However, while participation in such grand ventures is commendable, the true value of audit will only be realised if all clinicians feel involved. They must take part in discussions to plan projects, review results and improve practice locally. Clinicians should request information and advice from Clinical Effectiveness Department staff and guide the CGC in developing proposals for the annual audit programme.

\section{Assessing service quality}

Audit, research and development, consumer surveys and an efficient complaints procedure are not worth a handful of beans unless the standard of care improves where necessary. In our audit programme service managers will collect clinical outcomes data and highlight targets that have not been met. The analysis of adverse incident reports will identify areas that generate high levels of clinical uncertainty or accidents so that these may be considered for a risk management programme.
All of this information will be used by the CGC to help determine a comprehensive multidisciplinary (and sometimes, multi-agency) training programme. The training of individual staff is monitored by their line managers so that essential skdlls (e.g. resuscitation, knowledge of the Mental Health Act, psychosocial therapies and health and safety) are covered universally. More specific skills (e.g. neonatal care on the mother and baby unit) will be incorporated into the training grids for each clinical team. In this way, training will be determined by clinical activity and ultimately by the needs of patients, rather than random allocation to available courses.

In its White Paper The New NHS (Department of Health, 1997), the Government has stated clinical leadership to be the cornerstone of effective service delivery. This is not new. In psychiatry, the role of leader is enshrined in law for consultant psychiatrists as responsible medical officers, the duties of whom are listed by the relevant College statement (Royal College of Psychiatrists, 1996). Quality is not the sole domain of doctors: fellow professionals must also accept responsibility to meet standards. Quality objectives have been set for ward managers and community psychiatric nurses in York, and again it will be the responsibility of service managers to ensure that objectives are achieved.

Where performance is poor, organisations may expect no sympathy for failure to act if the safety of patients or the public is jeopardised. In a whistle-blowing climate, all NHS staff are encouraged to report concerns about colleagues. The CGC will identify major shortcomings and invite service managers and senior clinicians to consider ways of improving performance. A programme of remedial training may be involved. If this proves unsuccessful, it may become necessary to ask the head of service, medical director or chief executive to intervene as appropriate. The public will expect courageous and decisive action.

\section{What we want from the chief ezecutive}

A First Class Service (Department of Health, 1998) declares that a chief executive "carries ultimate responsibility for assuring the quality of services provided by the trust". So far I have described the role of the CGC, whose remit is limited to a distinct clinical area of manageable size. Professionals of all grades are required to monitor their own standards, but they also expect strong leadership from the top.

We look to the chief executive to ensure funding for access to information resources and training. The most difficult task will be to 
improve clinical performance of individuals or teams where results have fallen below acceptable standards. It is difficult to remove consultants, but the current medico-political climate demands such action in extreme circumstances. The General Medical Council (1998) has procedures to deal with "seriously deficient performance" under the Medical (Professional Performance) Act 1995 and there is talk of validating consultants every five years. Hopefully, the procedure described in the first part of this series, when applied with wisdom and sensitivity, will head off extreme situations. Problems will be effectively dealt with when they are small, in early career not left until there is no other remedy than retirement.

Patients will only benefit, however, if the relationship between workload and quality of care is recognised and addressed by chief executives. With so many vacant consultant posts nationally it is hard to envisage the solution to this puzzle. Perhaps a trust that is recognised for high standards may attract and keep good quality clinicians. The chief executive must strive to raise the quality profile of the trust in the public arena.

Clinicians should expect support and action from their chief executive when numbers of patients referred exceed resources available for reasonable quality of care. In forensic psychiatry, the shortage of secure hospital beds is beyond question. Refusal to accommodate individuals with personality disorders and sex offenders frequently causes ill feeling between clinicians, social services and the criminal justice agencies. Consultants and senior managers would benefit from the chief executive's support in defining and quantifying what the organisation can be expected to achieve. Limitations must be made explicit and adhered to otherwise quality will be sacrificed. This is where benchmark comparisons with other services can be helpful.

\section{Comment: top-down or bottom-up?}

Clinical governance, like many management initiatives, provokes discussion about ownership, organisational culture and the best ways to change behaviour. Many commentators appear to favour the bottom-up approach. However, total reliance on clinical staff to embrace the new culture, measure their own performance and challenge their peers to do likewise is probably naive. Previous behaviour suggests that many will not change. Clinical staff have no power to enforce widespread change within their organisation. Successful fulfilment of the ideals of clinical governance will require a combination of approaches. The shop floor must have a voice and will be aware of day to day problems associated with treating particular groups of patients. Change will only come about if clinicians contribute their expertise to the debate and find ways to improve. They will only be enthusiastic for issues that are directly relevant to their practice. However, unless training, audit and outcomes are monitored, the variation in performance between clinical teams will remain unacceptably high. Only firm leadership from above can provide the carrot and stick that may be required to guide the organisation forward.

Ultimately, the approach that our mental health directorate is taking will be judged on its results. For now, we aim to achieve a system of clinical governance that will deliver 'joined-up' quality. Joined-up quality may be accomplished if training, adverse incident reporting, user involvement, audit and operational policies are interlinked.

\section{References}

DEPARTMENT OF HEALTH (1997) The New NHS: Modem. Dependable. Cmnd 3807. London: The Stationery Office.

- (1998) A First Class Service: Quality in the New NHS. Leeds: Department of Health.

- \& HOME OFFICE (1992) Revlew of Health and Soctal Services for Mentally Disordered Offenders and Others Requiring Similar Services (The Reed Report). Cmnd 2088. London: HMSO.

FALLON, P.. Bluglass, R. EDWARDS, B., et al (1999) Report on the Committee of Inquiry into the Personality Disorder Unit at Ashworth Special Hospital (Volume 1). London: The Stationery Office.

GENERAL MEDICAL COUNCIL (1998) Maintaining Good Medical Practice. London: General Medical Council.

Grounds, A. (1997) Commentary on 'Inquiries Who Needs Them?' Psychiatric Bulletin, 21, 134-135.

Royal College of PSYChiatrists (1996) The Responsibilities of Consultant Psychiatrists. Revised Statement. Council Report CR51. London: Royal College of Psychiatrists.

SHEPPARD, D. (1996) Leaming the Lessons. Mental Health Inquiry Reports Published in England and Wales between 1969 and 1996 and their Recommendations for Improuing Practice (2nd edn). London: Zito Trust.

SNOWDEN, P. (1990) Regional Secure Units in England and Wales. In Principles and Practice of Forensic Psychiatry (eds R. Bluglass \& P. Bowden). pp. 1375-1386. London: Churchill Livingstone.

Jim Isherwood, Consultant Forensic Psychiatrist, Bootham Park, York YO30 7BY 